\title{
Generative Vermehrung von Amorphophallus johnsonii durch Handbestäubung
}

\author{
Margret Kiehlmann
}

\begin{abstract}
Amorphophallus johnsonii belongs to the Araceae family. Cultivation and generative propagation of Amorphophallus johnsonii by hand pollination with deep-frozen pollen is described.

\section{Zusammenfassung}

Amorphophallus johnsonii gehört zur Familie der Aronstabgewächse. Kultivierung und generative Vermehrung von Amorphophallus johnsonii durch Handbestäubung mit tiefgekühltem Pollen wird beschrieben.
\end{abstract}

\section{Die Gattung Amorphophallus}

Die Gattung Amorphophallus (Araceae) umfasst ca. 200 Arten aus den Tropen der Alten Welt von West-Afrika bis Polynesien. Die Arten sind terrestrisch und unverholzt, als Überdauerungsorgane werden Sprossknollen entwickelt. Meist treiben im Wechsel ein stark gefiedertes Laubblatt oder eine Infloreszenz aus (ITTENBACH 2003). Die im Palmengarten kultivierten Amorphophallus-Arten zeigen diesen Wechsel nicht zwangsläufig, manche Individuen bilden immer Blätter.

Die Knollen sind länglich, rundlich, elliptisch oder diskusförmig und je nach Art und Entwicklungszustand unterschiedlich groß. Nach einer bis zu mehreren Monaten dauernden Ruheperiode werden Wurzeln gebildet und entweder ein Blatt oder ein Blütenstand getrieben. Die Blätter betreiben Photosynthese, sodass energiereiche Vorratsstoffe (vor allem Stärke) in der Knolle eingelagert werden können. Amorphophallus bildet Wechselknollen, wobei sich neue Knollen über dem Rest der vergehenden vorjährigen Knolle entwickeln.

In der Regel wird zu einer Zeit nur ein Laubblatt mit einer dreiteiligen Spreite und einem kräftigen Blattstiel entwickelt. Der Blattstiel ist je nach Art unterschiedlich lang und dick sowie unterschiedlich gefärbt. An seiner Basis wird er

Abb. 1: Amorphophallus johnsonii, Teil der Spatha aufgeschnitten;

AP: Appendix, KA: Weibliche Blüten, PE: Blütenstandsstiel, SD: Kolben, SP: Spatha, ST: Männliche Blüten.

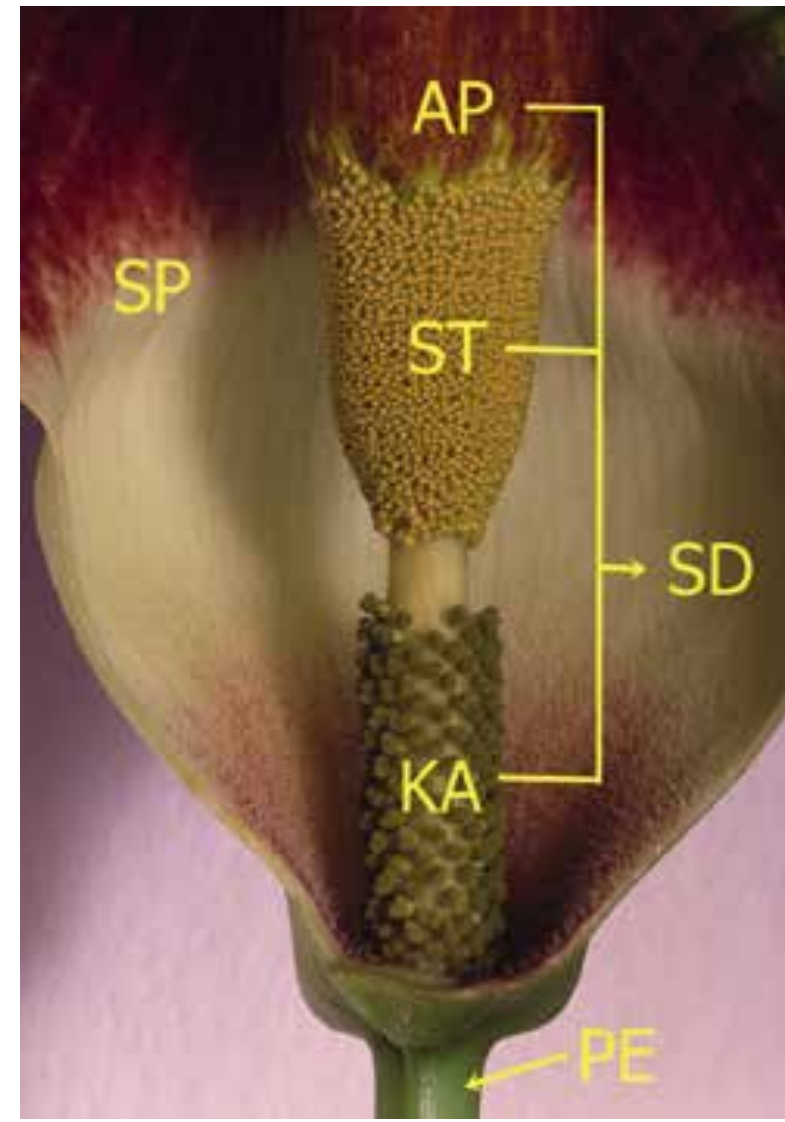

von 1-4 Niederblättern umgeben. Der Blütenstand ist je nach Art unterschiedlich lang gestielt, aber auch innerhalb einer Art variabel. Wenn die Pflanze von anderen höheren Pflanzen umgeben ist (auch in Kultur auf dicht bestückten Pflanztischen), wird der Stiel i. a. länger, um die Infloreszenz möglichst hoch über die anderen Pflanzen zu schieben.

Der Blütenstand gliedert sich in einen unverzweigten Kolben (Spadix) mit vielen unschein- 

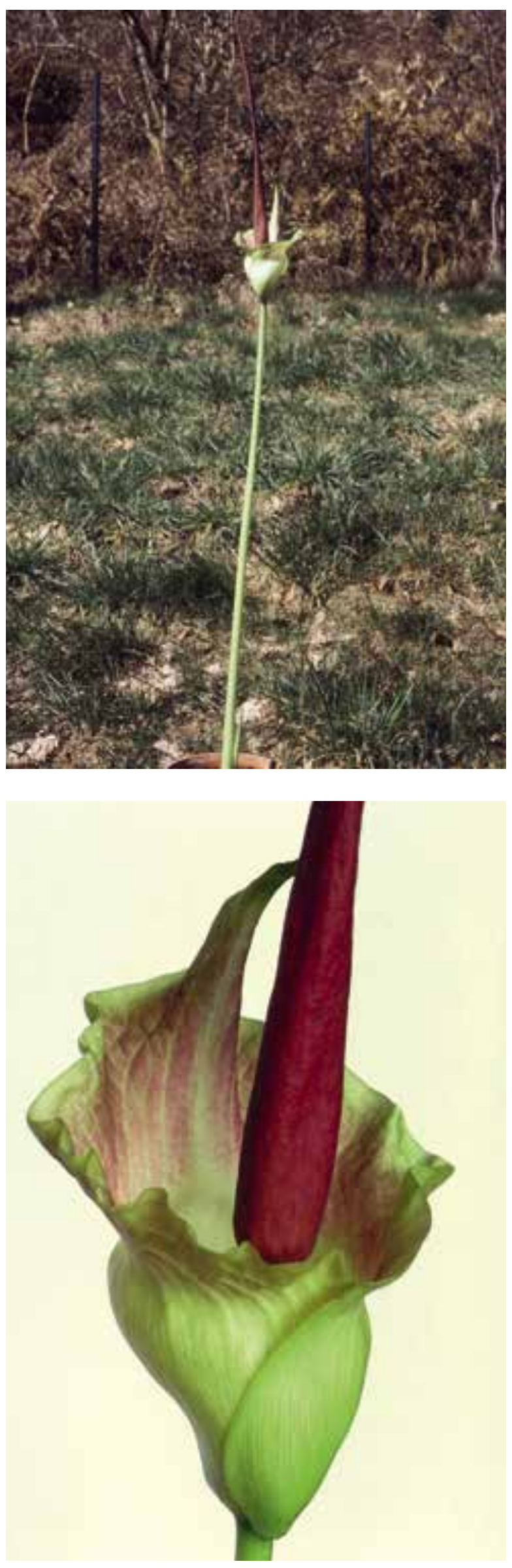

baren reduzierten Blüten und ein auffälliges Hochblatt (Spatha), das den Kolben umgibt und die Schauwirkung übernimmt. Die Blüten von Amorphophallus sind eingeschlechtlich, wobei die weiblichen Blüten sich am Grunde des Kolbens befinden. Darüber befindet sich die Zone mit männlichen Blüten, wobei bei manchen Arten weibliche und männliche Blüten durch eine sterile Zone voneinander getrennt sind (vgl. ItтEnbach 2003).

Der oberste sterile Bereich des Kolbens wird Appendix genannt, der wie der gesamte Kolben und die Spatha artabhängig von unterschiedlicher Gestalt, Farbe und Struktur ist. Er produziert Wärme und sorgt damit für eine bessere Verbreitung von Duftstoffen.

Amorphophallus-Arten blühen nur wenige Tage und sind vorweiblich (Bogner 1976, Beath 1991, Hetterscheid \& Ittenbach 1996). Die männlichen Blüten entlassen ihren Pollen erst 1-2 Tage nach der weiblichen Phase. Als Bestäuber dienen Insekten, die durch den Duft (für uns meist eher ein Gestank) des Blütenstandes angelockt werden. Jede Art hat einen spezifischen Geruch, z. B. nach Früchten, Schokolade, ranzigem Käse, Fäkalien, Fisch, Urin, Aas oder Petroleum (Kite et al. 1998), wodurch spezifische Bestäuber angelockt werden.

Der Fruchtstand ist je nach Art zylindrisch, konisch oder oval bis rund und trägt zahlreiche Beerenfrüchte. Diese sind rot, orange, selten blau oder weiß gefärbt und enthalten 1-4 Samen. Innerhalb der Gattung Amorphophallus gibt es auch einige Arten, die sich vegetativ durch Tochter- und Brutknollen fortpflanzen sowie apomiktische Arten, die Samen ohne Bestäubung bilden (z. B. A. bulbifer, A. muelleri, A. longiconnectivus und $A$. kiusianus; Claudel \& Galloway 2012).

Amorphophallus-Arten haben ein weites Größenspektrum. Die größte Art ist die bekannte Titanenwurz (Amorphophallus titanum) aus Su-

Abb. 2 (oben): Infloreszenz von Amorphophallus johnsonii.

Abb. 3 (unten): Teilansicht der Infloreszenz von Amorphophallus johnsonii. 


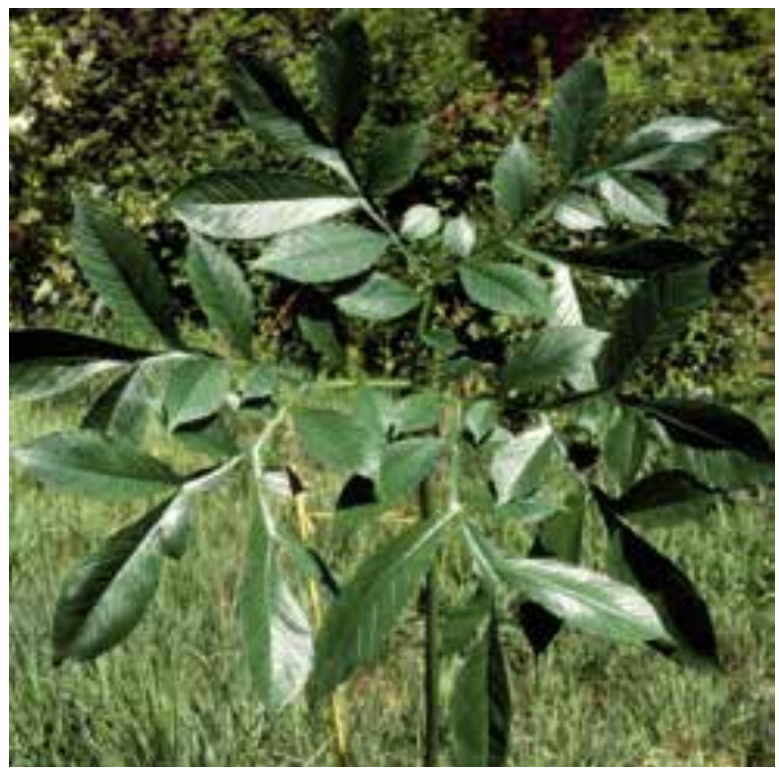

matra, deren Knolle über $75 \mathrm{~kg}$ Gewicht erreicht und die ein bis zu $5 \mathrm{~m}$ hohes Blatt mit einer $7 \mathrm{~m}$ breiten Spreite entwickeln kann (Hetterscheid \& Ittenbach 1996). Bei kräftigen Exemplaren wird der Blütenstand über $3 \mathrm{~m}$ hoch und $1,50 \mathrm{~m}$ breit, er ist damit der größte unverzweigte Blütenstand, also die größte Blume, im Pflanzenreich.

\section{Amorphophallus jobnsonii}

Diese Art ist in West-Afrika (Guinea, Liberia, Elfenbeinküste, Ghana, Burkina Faso, Mali) heimisch. Sie wächst in der Savanne, im Galeriewald und auf Inselbergen. Unter Savannenbedingungen entwickelt $A$. johnsonii einen langen Blütenstandsstiel, während dieser bei Individuen im Wald deutlich kürzer ausfällt (Hetterscheid \& Ittenbach 1996). Zwischen beiden Extremformen gibt es vielfältige Übergänge. Amorphophallus johnsonii kommt in Höhen bis $600 \mathrm{~m}$ vor, blüht von (Januar-) März bis Juni und fruchtet von April bis August (ItтenbaCh 2003). In Kultur kann die Art beachtliche Größen erreichen, das größte Blatt in Kultur der Autorin hatte einen Blattstiel von $142 \mathrm{~cm}$ Länge und eine Blattspreite von $132 \mathrm{~cm}$

Abb. 4 (links): Blatt von Amorphophallus johnsonii.

Abb. 5 (rechts): Knolle von Amorphophallus johnsonii mit Resten der vorjährigen Knolle.

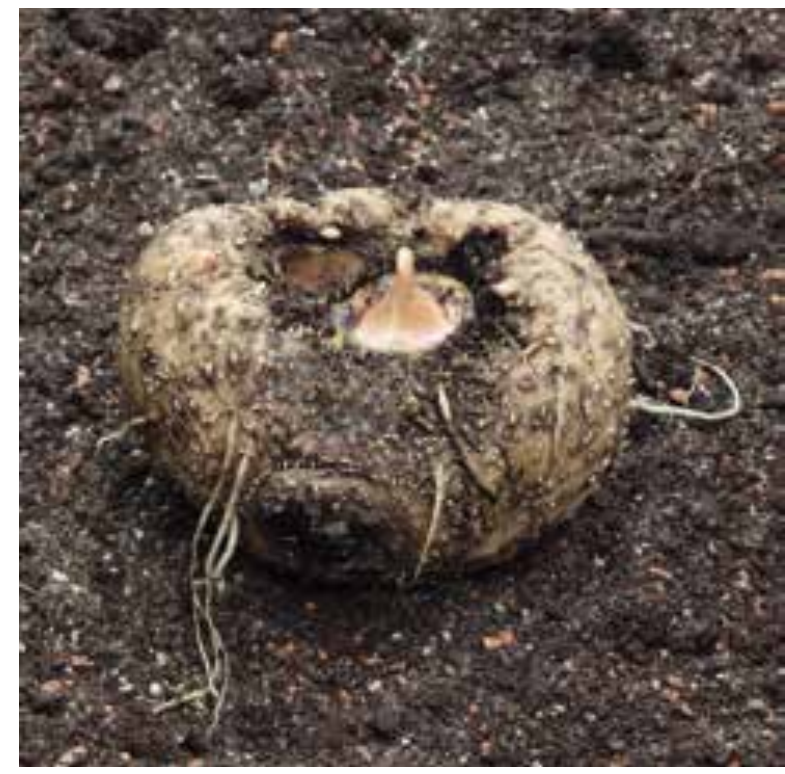

Durchmesser. Eine Infloreszenz erreichte eine Höhe von $221,5 \mathrm{~cm}$, wobei der Blütenstandsstiel $152 \mathrm{~cm}$ betrug und die Blütenstandsachse 69,5 cm maß. Die schwerste Knolle wog 1620 g. Bei den afrikanischen Amorphophallus-Arten entwickelt sich im Gegensatz zu den meisten asiatischen Arten gleichzeitig mit der Infloreszenz ein neues Blatt.

\section{Standort, Pflege und Pollenlagerung}

Im Juli 1995 erhielt die Autorin von der Elfenbeinküste zwei runde Knollen mit einem Durchmesser von $2 \mathrm{~cm}$. Seit dieser Zeit sind sie bei ihr erfolgreich in Kultur. Der Standort ist ein Südfenster in einem Raum mit Fußbodenheizung und Tagestemperaturen je nach Jahreszeit von $20-30^{\circ} \mathrm{C}$, die Nachttemperaturen liegen um die $20^{\circ} \mathrm{C}$ bei einer relativen Luftfeuchtigkeit zwischen 45 und $65 \%$. Ausreichende Wärme ist generell für AmorphophallusArten u.a. auch für das Wurzelwachstum wichtig. Während die Knollen von $A$. konjac zur Blütezeit mitunter wurzellos sind, sind dann die Knollen von A. titanum bewurzelt, wobei die Wurzeldichte von der Temperatur abhängig ist. In Kultur sollte auf ein stabiles Wurzelwerk Wert gelegt werden, da sonst der mechanische Halt fehlt.

Die Pflanzerde besteht aus einer Mischung handelsüblicher Blumenerde, Sand und Bims im Verhältnis 3:1:0,5. Auch wenn die vegetative 

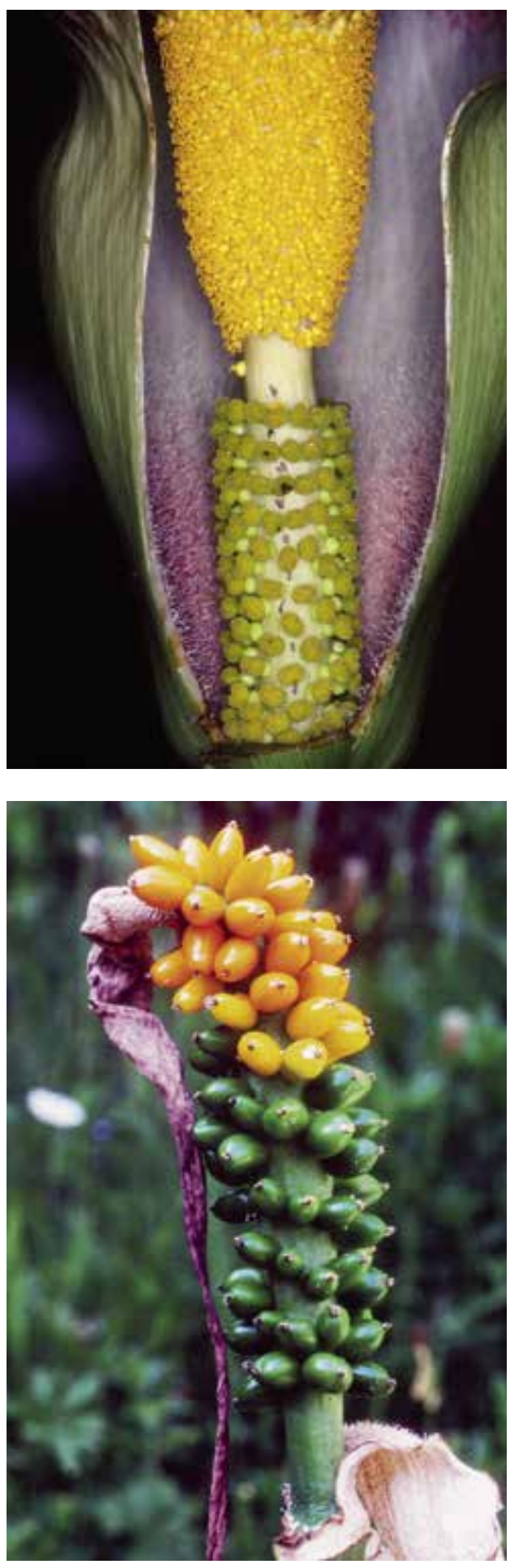

Vermehrung von $A$. johnsonii kein Problem ist, hat die Autorin besonderes Interesse an der generativen Vermehrung ihrer AmorphophallusArten.

Da die weiblichen Blüten empfängnisbereit sind, bevor der eigene Pollen reif ist, kann die Pflanze sich nicht selbst bestäuben. Um eine erfolgreiche Bestäubung von Hand in Kultur zu erreichen, muss man um einen Tag versetzt zwei blühende Exemplare haben oder Pollen verwenden, der im Kühlschrank oder in der Tiefkühltruhe aufbewahrt wurde. In den Botanischen Gärten Bonn wird letztgenannte Methode seit Jahren praktiziert (HETTERsCHeID et al. 1998). Auch der Autorin ist es bereits mehrfach gelungen, $A$. johnsonii mit Hilfe von gekühltem Pollen erfolgreich zu bestäuben und zu vermehren. Verwendet wurde dabei Pollen, der bis zu 15 Tage lang im Kühlschrank bei $3-6^{\circ} \mathrm{C}$ gelagert wurde. Einmaligen Erfolg gab es auch mit bei $-18^{\circ} \mathrm{C}$ tiefgekühltem Pollen (Lagerzeit 2 Monate, 21 Tage, s. u.). Die längste Speicherzeit von tiefgekühltem Pollen aller bisher von ihr kultivierten Amorphophallus-Arten, mit denen die Autorin fertile Samen erzeugte, betrug bei A. mossambicensis 30 Monate und 22 Tage (vgl. auch Kiehlmann \& Kiehlmann 2003).

\section{Blühvorgang, Bestäubung per Hand und Einsammeln des Pollens}

Am 17.9. 2008 wurde eine $516 \mathrm{~g}$ schwere Knolle von $A$. johnsonii ausgetopft und bei Zimmertemperatur trocken gelagert. Am 9.12.2008 wurde die jetzt noch $457 \mathrm{~g}$ schwere Knolle, die bereits einen spitzen kleinen Austrieb zeigte, eingesetzt. Ende Februar 2009 schob sich die noch eingerollte, grüne Spatha aus dem 3. Niederblatt. Wenige Tage vor der eigentlichen Blühperiode wurde die Spatha farbig und bau-

Abb. 6 (oben): Pollenschüttung bei Amorphophallus johnsonii, Markierungen zur Unterscheidung der Bestäubung mit Pollen unterschiedlicher Lagerungszeiten.

Abb. 7 (unten): Fruchtstand von Amorphophallus johnsonii mit Beeren in verschiedenen Reifestadien.

Abb. 8 (Seite 21): Fruchtstand mit reifen Beeren. 


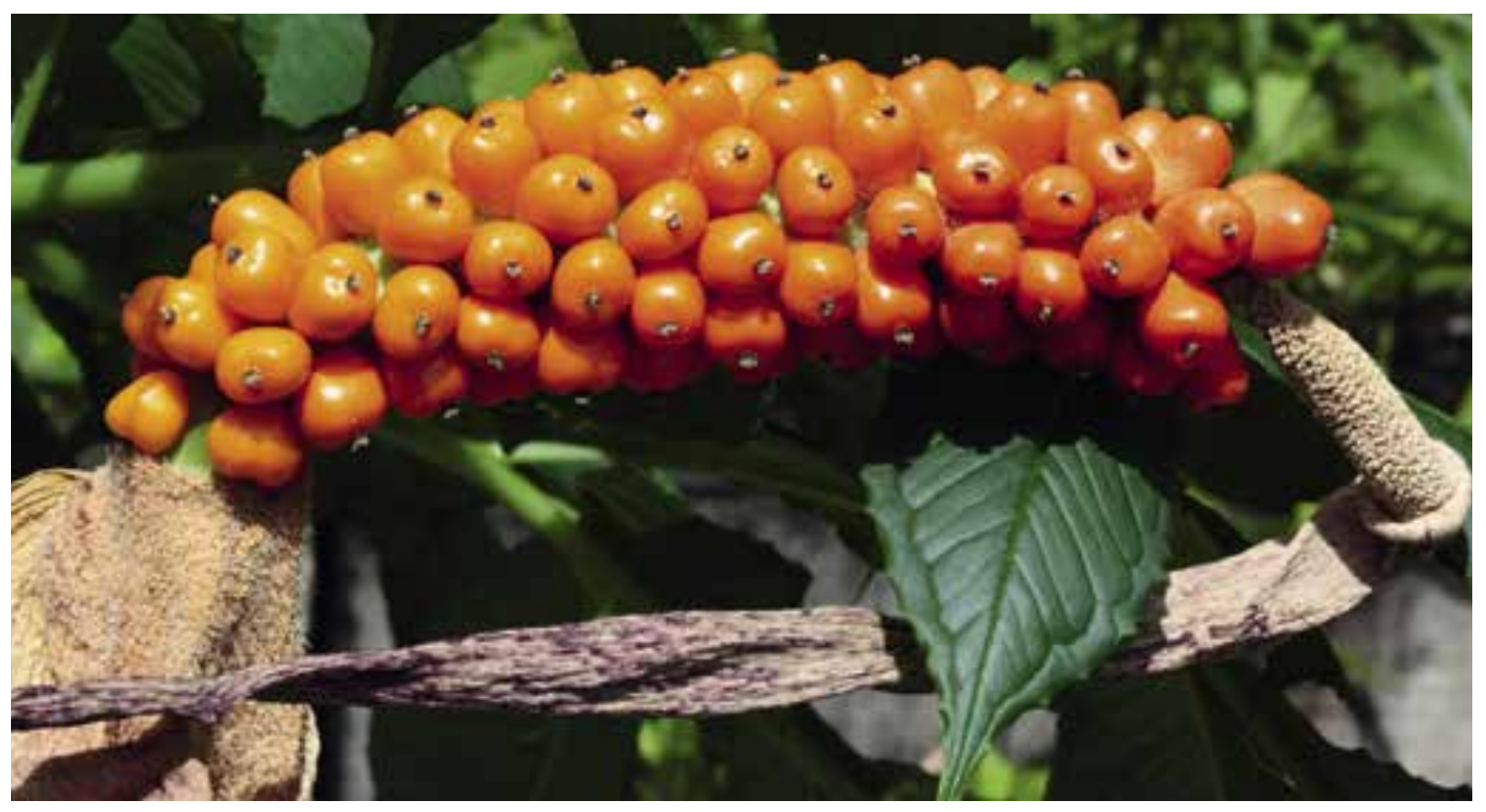

chig, öffnete sich leicht und es erschien eine Einschnürung. In den Morgenstunden des 12.3.2009 war die Spatha vollständig geöffnet und entsandte einen Geruch nach Aas mit Schwefelkomponenten, der sich bis zum Abend noch verstärkte.

Nun wurde der Pollen der Tiefkühltruhe entnommen und in einem geschlossenen Eppendorff-Gefäß $(0,5 \times 3,5 \mathrm{~cm})$ in einer halben Stunde auf Zimmertemperatur gebracht. Der Pollen stammte von einem zweiten Exemplar, das über zwei Monate zuvor geblüht hatte (s. o.), und war vor dem Einfrieren zwei Tage lang über Silikagel getrocknet worden. Inzwischen wurde die Spatha im vorderen Teil aufgeschnitten, um die zu bestäubenden weiblichen Blüten besser erreichen zu können. Der Pollen wurde mittels eines kleinen Pinsels auf die feuchten Narben gebracht.

Die Pollenschüttung erfolgte einen Tag nach der intensiven Geruchsentwicklung gegen $20 \mathrm{Uhr}$, wobei die Spatha sich bereits etwas zusammengezogen hatte. Gelber, klebriger Pollen wurde in Schnüren aus den Antheren entlassen. Der Blütenduft war nun zwiebelähnlich. Für das Einsammeln des Pollens wurde ein trockenes Eppendorff-Gefäß bereitgehalten. Mit einem kleinen Spatel wurde der Pollen vorsichtig von den Antheren abgeschabt und in das Gefäß ge- schoben. Dieses wurde mit geöffnetem Verschluss in eine Tablettendose gelegt, in deren Deckel sich ein Trockenmittel (Silikagel) befand.

\section{Pollenlagerung}

Je nach Feuchtigkeit des Pollens bleibt das geöffnete Gefäß mit dem Pollen für 1-2 Tage in der Tablettendose. Danach wird es herausgenommen, sofort verschlossen und entweder bei $3-6^{\circ} \mathrm{C}$ im Kühlschrank gelagert oder bei $-18^{\circ} \mathrm{C}$ tiefgekühlt.

\section{Samenernte, Aussaat, Sämlinge}

Wenn die Bestäubung erfolgreich war, bilden sich aus den Fruchtknoten zunächst grüne, gegen Ende der 3-4-monatigen Reifezeit orangefarbene, rund bis oval geformte Beeren. Sobald diese weich werden oder bei Berührung abfallen, werden sie geerntet.

Das im Kap. 4 beschriebene, handbestäubte Exemplar lieferte 134 Beeren, von denen die Hälfte nur taube Samen enthielt. Die fertilen Beeren brachten ein durchschnittliches Gewicht von $485 \mathrm{mg}$ auf die Waage, die 5,6 $8 \mathrm{~mm}$ großen Samen wogen durchschnittlich $149 \mathrm{mg}$.

Zur Vorbereitung der Aussaat wird das Fruchtfleisch entfernt, die Samen 2-3 Tage gewässert und dann in eine Mischung aus Blu- 


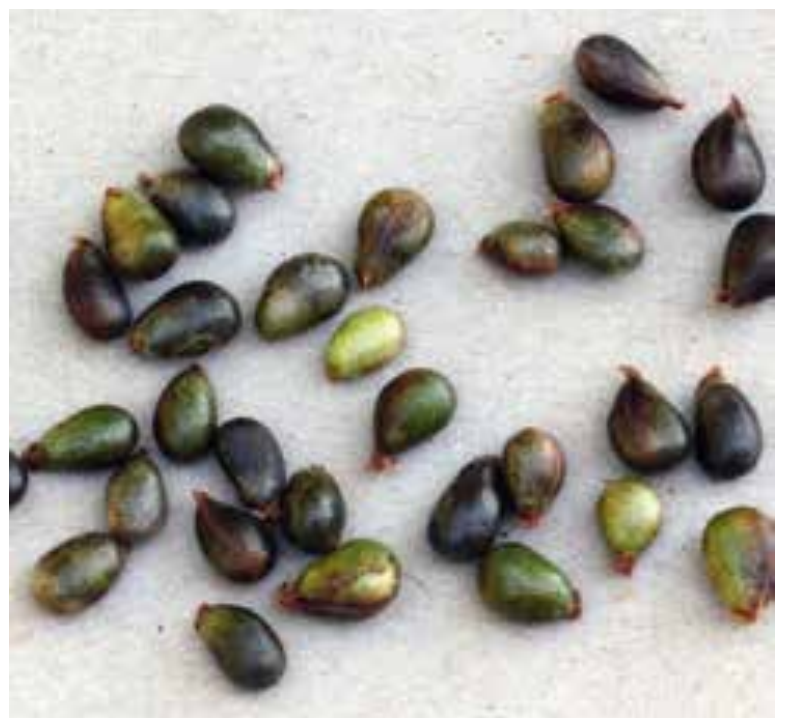

menerde und Sand (Mischungsverhältnis 1:1) gepflanzt. Nach 7-10 Monaten erscheinen die Sämlinge.

\section{Länge der Keimfähigkeit des Pollens von Amorphophallus johnsonii}

Von Beobachtungen an Amorphophallus johnsonii im Regenwald von Ghana ist bekannt, dass während der weiblichen Phase von $A$. johnsonii durch die Geruchsentwicklung hauptsächlich Aaskäfer (Phaeochrous amplus) angelockt werden (Bеath 1991). Während der Blühphase von Aronstabgewächsen kommen aber nicht nur mögliche Bestäuber, sondern auch andere Insekten auf der Suche nach Futter in den Blütenstand (Gibernau 2003). Nicht eine einzige Araceen-Art ist bekannt, die Nektar als Belohnung anbietet (SCHWERDTFEgER et al. 2002).

Eine erfolgreiche Befruchtung findet nur statt, wenn Pflanzen in der weiblichen Phase am selben Abend von den Käfern besucht wurden, die von einer Pflanze in der männlichen Phase kamen. Das deutet auf eine kurze Lebensdauer des Pollens hin. Da die Pollenkörner von A. johnsonii sehr dünnwandig sind und schnell austrocknen können, ist der Zeitraum ihrer Keimfähigkeit sehr kurz. Dem Pollen mancher Arten der Unterfamilie Aroideae, zu der auch Amorphophallus gehört, fehlt das Sporopollenin (PanuschKa 2009), stattdessen enthält die Oberfläche dieser Pollenkörner Polysaccharide, die eine wesentlich kürzere Lebensdauer als das Sporopollenin auf-

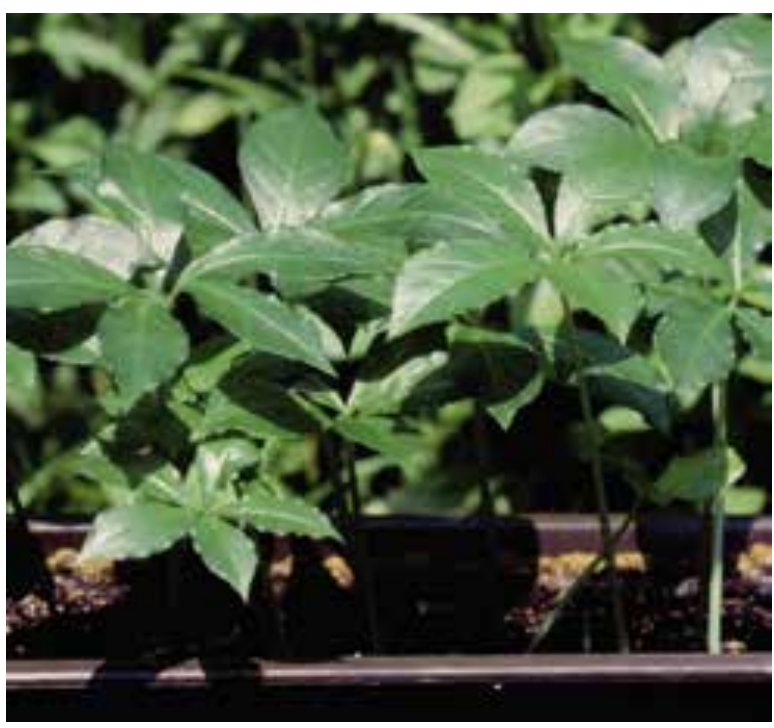

weisen und vermutlich die Länge der Keimfähigkeit verringern. Dies mag ein Grund dafür sein, dass die Vermehrung von $A$. johnsonii mit länger gelagerten Pollen seltener gelingt.

Versuche haben bestätigt, dass ein funktioneller Zusammenhang zwischen kurzer Bestäubungszeit und Instabilität der Pollenoberfläche besteht. So fehlte sichtbarer Pollen an markierten und wiedergefangenen Käfern, die die Infloreszenzen 1-2 Tage vorher besucht hatten, d. h. der Pollen wurde bereits unmittelbar nach dem ersten Blütenbesuch auf den Narben einer anderen Infloreszenz abgestreift (BEATH 1991).

\section{Schlussbetrachtung}

Nicht immer gelingt eine Handbestäubung, wenn z. B. die Pflanzen selbststeril sind oder der Pollen zu kurzlebig ist und keine zweite blühende Infloreszenz in den nächsten Stunden verfügbar ist. Misserfolge können durch zu trockene oder zu feuchte Lagerung des Pollens entstehen oder

Abb. 9 (links): Samen von Amorphophallus johnsonii.

Abb. 10 (rechts): Sämlinge.

Abb. 11 (Seite 23 oben): Amorphophallus abyssinicus ist eine andere afrikanische Art, die in ähnlichen Lebensräumen wie Amorphophallus johnsonii vorkommt. Auch sie blüht regelmäßig in Kultur.

Abb. 12 (Seite 23 unten): Amorphophallus titanum im Palmengarten, die größte und bekannteste Art der Gattung. 
wenn die Narben zum Zeitpunkt der Bestäubung bereits ihre Empfängnisfähigkeit verloren haben. Bei $A$. johnsonii sind das Anschwellen der meisten Fruchtknoten nach 8-14 Tagen und ein druckfester Infloreszenzstiel gute Anzeichen für eine erfolgreiche Befruchtung. Während bei anderen Arten mit kurzem Blütenstiel, wie z. B. bei $A$. lewallei und A. mossambicensis, sich dieser bei erfolgreicher Befruchtung schnell verlängert, dauert das Wachstum des Stiels bei A. johnsonii nach gelungener Befruchtung wesentlich länger. Auf jeden Fall ist es extrem spannend, damit $\mathrm{zu}$ experimentieren, unter welchen Bedingungen eine Handbestäubung von Erfolg ist.

\section{Literatur}

Beath, D. N. 1996: Pollination of Amorphophallus johnsonii (Araceae) by carrion beetles (Phaeochrous amplus) in a Ghanaian rain forest. - J. Trop. Ecol. 12: 409-418. Bogner, J. 1976: Amorphophallus maculatus N. E. BR. - Palmengarten 1976/3: 83-87.

Gibernau, M. 2003: Pollinators and visitors of aroid inflorescences. - Aroideana 26: 66-83.

Hetterscheid, W., Koenen, M., Lobin W., Ittenbach, S. \& Neumann, M. 1998: Amorphophallus titanium in cultivation. - In: Barthlott, W. \& LoBin, W. (Hrsg) : Amorphophallus titanum. - Trop. Subtrop. Pflanzenwelt 99: $198-205$.

Claudel, C. \& Galloway, A. 2012: Hybridization of Amorphophallus - state of the art. - Aroideana 35: 103-108. Hetterscheid, W. \& Ittenbach, S. 1996: Everything you always wanted to know about Amorphophallus, but were afraid to stick your nose into!!! - Aroideana 19: 7-131. ItTenbach, S. 2003: Revision der afrikanischen Arten der Gattung Amorphophallus (Araceae). - Englera 25: 1-263.

Kiehlmann, M. \& Kiehlmann, D. 2003: Propagation of some aroids by hand pollination with stored pollen. - Aroideana 26: 56-65.

Kite, G. C., Hetterscheid, W. L. A., Lewis, M. J., Boys, P.C., Ollerton, J., Cocklin, E., Diaz, A. \& Simmonds, M. S. J. 1998: Inflorescence odours and pollinators of Arum and Amorphophallus (Araceae). - In: Owens, S. J. \& Rudall, P. J.: Reproductive biology. - Kew. Panuschka, C. 2009: Pollen im Polysaccharid-Mantel. Laborjournal 1/2: 33-35.

Schwerdtfeger, M., Gerlach, G. \& Kaiser, R. 2002: Anthecology in the neotropical genus Anthurium (Araceae) - a preliminary report. - Selbyana 23: 258-267.

\section{Dank}

Herzlicher Dank gilt Frau Stefanie Weber, Amorphophallus-Kultivateurin im Palmengarten, für Durchsicht des Manuskriptes und Ergänzungen.
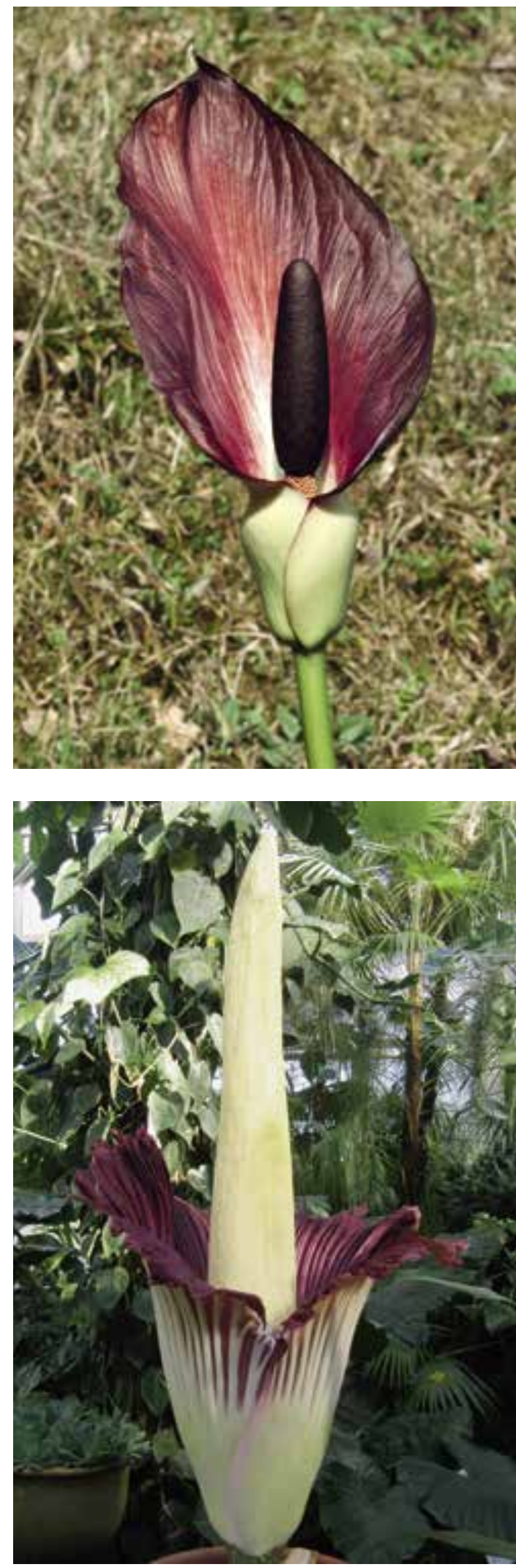